\title{
Principales motivos de consulta externa ortopédica en un hospital de segundo nivel en México
}

\section{Main causes of orthopedic external consultation in a Second Level Clinic in Mexico}

\author{
Santos-Zaldivar KP, ${ }^{*}$ Erosa-Villarreal RA ${ }^{\ddagger}$ López-Muñoz R, ${ }^{\S}$ Méndez-Domínguez N`
}

Escuela de Medicina. Universidad Marista de Mérida.

RESUMEN. Introducción: La epidemiología de las enfermedades permite la generación de medidas preventivas para la modificación de su evolución natural o para evitar su aparición. En los hospitales de segundo nivel de atención se tratan la mayor parte de las patologías de cada especialidad médica. Por lo tanto, saber los principales motivos de consulta externa en ortopedia y su predominio estacional es una herramienta para la implementación de estrategias en beneficio de los pacientes. Material y métodos: Estudio observacional realizado con la población de pacientes del servicio de consulta externa de ortopedia de un hospital de segundo nivel de atención de Enero a Diciembre del año 2017. Se incluyeron pacientes mayores de 18 años que acudieron a consulta por primera vez. Resultados: De 11,704 consultas otorgadas en el período, 7,862 (67.17\%) fueron mujeres y 3,842 (32.82\%) hombres. La edad promedio de los pacientes fue de $51.84 \pm 0.14$. La causa más frecuente de consulta fueron las artropatías (47.65\%). Durante el otoño se otorgó el mayor número de consultas (32.33\%). Conclusiones: Las artropatías, específicamente la gonartrosis, fue el principal motivo de consulta externa, representando casi la mitad de las consultas otorgadas. Es transcendental identificar los factores de riesgo modificables de estas patologías con el objetivo de disminuir el impacto negativo que genera a nivel funcional, económico y de calidad de vida. Importancia clínica: La realización de más estudios epidemiológicos sobre las patologías ortopédicas permitirá mejo-
ABSTRACT. Introduction: The epidemiology of diseases allows the generation of preventive measures, for the modification of their natural evolution or to prevent their appearance. In second-level hospitals of care, most of the pathologies of each medical specialty are treated. Therefore, knowing the main reasons for external consultation in orthopedics and their seasonal predominance is a tool for implementing strategies for the benefit of patients. Material and methods: Observational study carried out with the patient population of the external orthopedic consultation service of a second-level hospital of care, from January to December 2017. Patients over the age of 18 who first attended consultation were included. Results: 11,704 consultations granted in the period, 7,862 (67.17\%) women and 3,842 (32.82\%) men. The average age of patients was $51.84 \pm 0.14$. The most common cause of consultation was arthropathies (47.65\%). During the autumn, the largest number of consultations (32.33) were granted. Conclusions: Arthropathies, specifically gonarthrosis, were the main reason for external consultation, accounting for nearly half of the consultations granted. It is transcendental to identify the modifiable risk factors of these pathologies with the aim of reducing the negative impact it generates at the functional, economic and quality of life level. Clinical relevance: Further epidemiological studies on orthopedic pathologies will improve preventive strategies and optimize resources to improve patient care.

\section{Nivel de evidencia: III}

* Médico interno de pregrado. Escuela de Medicina. Universidad Marista de Mérida.

* Médico cirujano.

$\S$ Cirujano Ortopedista. Profesor Titular de la Materia de Ortopedia Universidad Marista de Mérida.

" Médico cirujano. Maestra en Ciencias. Doctora en Ciencias de la salud. Maestra en Bioética. Profesor Titular de las asignaturas:

Medicina Preventiva, Métodos clínicos y epidemiológicos y Epidemiología Clínica Universidad Marista de Mérida.

Correspondencia:

Ricardo López Muñoz

Clínica de Mérida Calle 32 Núm. 242, García Ginerés, C.P. 97070, Mérida, Yucatán.

E-mail: ricardo@drhombro.com

Citar como: Santos-Zaldivar KP, Erosa-Villarreal RA, López-Muñoz R, Méndez-Domínguez N. Principales motivos de consulta externa ortopédica en un hospital de segundo nivel en México. Acta Ortop Mex. 2020; 34(5): 303-308. https://dx.doi.org/10.35366/97993

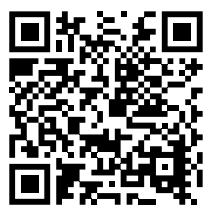


rar las estrategias preventivas y optimizar los recursos para mejorar la atención de los pacientes.

Palabras clave: Consulta externa, primera vez, artropatías, gonartrosis, epidemiología.

\section{Introducción}

La traumatología y ortopedia es una especialidad médico quirúrgica donde se tratan las alteraciones del sistema musculoesquelético, ya sea a nivel óseo o de los tejidos blandos, que provoca discapacidad funcional por dolor o deformidad. En esta especialidad las patologías se pueden abordar desde la sala de urgencias o desde la consulta externa y el tratamiento puede variar entre conservador o quirúrgico.

El sistema de salud en México se conforma por tres niveles, el primer nivel es el encargado de resolver las necesidades básicas de la población y actividades que pueden ser resueltas con promoción de la salud. En el segundo nivel de atención se encuentran los hospitales y establecimientos donde se prestan servicios de las especialidades y se resuelven hasta $95 \%$ de los problemas de salud. El tercer nivel de atención se encarga del manejo de patologías complejas que requieren procedimientos especializados y de alta tecnología. ${ }^{2}$

Para fines de este estudio nos centraremos en el segundo nivel de atención, donde son referidas y atendidas la mayor parte de las patologías ortopédicas. Es importante considerar que las patologías ortopédicas generan una alta demanda en la consulta de los hospitales generales. El retraso en el diagnóstico y/o tratamiento de dichas patologías tiene implicaciones significativas en la calidad de vida de los pacientes así como una carga económica importante para el sector salud debido al ausentismo laboral que generan y el costoso tratamiento de las complicaciones. Se considera que es la condición médica que produce mayor incapacidad en términos de morbilidad y economía. ${ }^{1,3,4}$

Esto no sólo plantea un problema de salud, sino que conlleva a otras repercusiones debido a los costos directos e indirectos que ocasiona, aunado al inconveniente que representa para la competitividad y la eficiencia de empresas, organizaciones y administraciones públicas, además de tener repercusiones sicosociales y familiares. ${ }^{1,3}$

A nivel internacional los trastornos musuculoesqueléticos tienen una alta incidencia y prevalencia en la población con actividad laboral, la cual va en aumento, lo que genera un impacto socioeconómico importante. ${ }^{3,5} \mathrm{El}$ objetivo de este estudio es describir los principales motivos de consulta externa en ortopedia en un hospital de segundo nivel en 2017 así como determinar el predominio estacional de las patologías.

\section{Material y métodos}

El estudio que se presenta corresponde a un diseño observacional descriptivo de corte transversal retrospectivo. La
Key words: External consultation, arthropaties, gonarthrosis, epidemiology.

población del estudio estuvo conformada por los pacientes que acudieron al Servicio de Consulta Externa Ortopédica del Hospital General de México SS de Enero a Diciembre de 2017 que se encontraban registrados en la base de datos de acceso abierto del hospital.

Se incluyó a los pacientes de primera vez con edad igual o mayor de 18 años. Se excluyó del estudio a la población pediátrica, registros pertenecientes a consultas subsecuentes, pacientes con patología ortopédica y/o traumatismo que fueron atendidos y tratados en otros servicios del hospital. Se eliminaron los pacientes cuyo diagnóstico y codificación de CIE-10 no correspondía a patologías ortopédicas ni su fisiopatología tenía relación con el desarrollo de alguna complicación ortopédica A49, T795, S210, C108, E105, E108, L921. E249, N171, N111. También se eliminaron casos únicos con diagnóstico y codificación CIE-10 de origen congénito Q669, Q655. Por último, se eliminaron 49 consultas otorgadas en Diciembre de 2016 registradas en la base de datos debido a que no pertenecían al período de tiempo estudiado.

La unidad observacional fue cada consulta externa ortopédica perteneciente a nuestra población de estudio, teniendo como variable dependiente el diagnóstico registrado en la base de datos codificado según el CIE-10 (nominal). En relación a las variables independientes se establecieron las siguientes: edad (numérica: discreta), grupo etario (categórica: adulto joven, adultez y adulto mayor); sexo (binaria: masculino/femenino) y estacionalidad (nominal: invierno, primavera, verano y otoño).

La selección de las fuentes, métodos, técnicas y procedimientos de recolección de la información: Los datos se obtuvieron de la base «Consultas otorgadas de 2017» de libre acceso del hospital en estudio. Se filtraron las consultas por especialidad utilizando «ortopedia» como palabra clave, con los resultados obtenidos se procedió a la creación de una nueva base empleando el software Excel, posteriormente se realizó un filtrado por tipo de consulta, seleccionado sólo a los pacientes de primera vez, de los cuales se realizó una última selección por edad, siendo de 18 años en adelante el rango utilizado. Finalmente se procedió a codificar y analizar los datos en el programa estadístico Stata 14.

\section{Resultados}

En total se registraron 11,704 consultas externas ortopédicas en el período de Enero a Diciembre de 2017, de las cuales $7,862(67.17 \%)$ fueron pacientes femeninos y 3,842 $(32.82 \%)$ masculinos. En un rango de edad de 18 a 105 años. La edad promedio fue de $51.84 \pm 0.14$ (Tabla 1). 
Con respecto a los resultados por grupo etario, se tuvieron 2,804 (24\%) adultos jóvenes (de 18 a 40 años), de los cuales $1,460(12.5 \%)$ fueron mujeres y $1,344(11.5 \%)$ hombres. El grupo de la adultez (de 41 a 60 años) conformaron $6,107(52 \%)$ de las consultas otorgadas, de las cuales 4,642 $(40 \%)$ fueron otorgadas a mujeres y $1,465(12 \%)$ a hombres y finalmente los adultos mayores fueron 2,793 (24\%), 1,760 $(15 \%)$ mujeres y $1,033(9 \%)$ hombres.

El principal motivo de consulta externa lo conformaron las artropatías con 5,581 registros, lo que corresponde a $47.65 \%$, seguidas de las dorsopatías con 2,298 (25.61\%) y en tercer lugar se encuentran los trastornos de los tejidos blandos con 829 (7.08\%) consultas (Tabla 2).

La artropatía con mayor incidencia fue «otras gonartrosis primarias» con 2,319 pacientes, lo cual representa $41.55 \%$ de este grupo. El segundo y tercer lugar de las artropatías con mayor incidencia lo ocuparon respectivamente la osteoartrosis primaria generalizada con $1,718(30.78 \%)$ y otras coxartrosis primarias con 541 (9.69\%). En $88.48 \%$ de la consulta externa perteneciente a las artropatías lo conformaron las 10 más frecuentes de este grupo (Tabla 3).

Prácticamente se respetó el patrón de frecuencia en los resultados por grupo etario, siendo las artropatías la principal patología abordada en la consulta externa ortopédica en los grupos adulto joven, adultez y adulto mayor (Tabla 4); sin embargo, en el grupo de adulto joven el tercer lugar lo

\begin{tabular}{|lccc|}
\hline \multicolumn{4}{|c|}{ Tabla 1: Características sociodemográficas. } \\
\hline & & $\mathrm{n}(\%)$ & {$[$ [nt. Conf. 95\%] } \\
\hline Edad & $51.84 \pm 0.14$ & $11,704(100.00)$ & {$[51.55-52.14]$} \\
Masculino & $48.36 \pm 0.28$ & $3,842(32.82)$ & {$[47.81-48.91]$} \\
Femenino & $53.55 \pm 0.17$ & $7,862(67.17)$ & {$[53.21-53.88]$} \\
\hline
\end{tabular}

Tabla 2: Principales motivos de consulta externa ortopédica.

\begin{tabular}{|c|c|c|}
\hline Motivos de consulta & $\mathrm{n}(\%)$ & [Int. Con. 95\%] \\
\hline 1. Artropatías & $5,581(47.65)$ & {$[0.467-0.485]$} \\
\hline 2. Dorsopatías & $2,298(25.61)$ & {$[0.248-0.264]$} \\
\hline $\begin{array}{l}\text { 3. Trastornos de los tejidos } \\
\text { blandos }\end{array}$ & $829(7.08)$ & {$[0.066-0.075]$} \\
\hline $\begin{array}{l}\text { 4. Traumatismos en rodillas } \\
\text { y piernas }\end{array}$ & $599.24(5.12)$ & [0.047-0.055] \\
\hline $\begin{array}{l}\text { 5. Traumatismos de codo y } \\
\text { del antebrazo }\end{array}$ & $409.64(3.50)$ & {$[0.031-0.038]$} \\
\hline $\begin{array}{l}\text { 6. Traumatismos en las } \\
\text { muñecas y manos }\end{array}$ & $335.90(2.87)$ & {$[0.025-0.031]$} \\
\hline $\begin{array}{l}\text { 7. Traumatismos de los } \\
\text { hombros y brazos }\end{array}$ & $316.00(2.70)$ & [0.024-0.030] \\
\hline $\begin{array}{l}\text { 8. Traumatismos en tobillos } \\
\text { y pies }\end{array}$ & $218.86(1.87)$ & [0.016-0.021] \\
\hline $\begin{array}{l}\text { 9. Traumatismos en caderas } \\
\text { y muslos }\end{array}$ & $117.04(1.00)$ & [0.008-0.012] \\
\hline $\begin{array}{l}\text { 10. Trastornos de los } \\
\text { nervios, de las raíces y } \\
\text { de los plexos nerviosos }\end{array}$ & $98.31(0.84)$ & [0.006-0.010] \\
\hline
\end{tabular}

Tabla 3: Principales artropatías.

$\begin{array}{lc}\text { Patología } & \mathrm{n}(\%) \\ \text { 1. Otras gonartrosis primarias } & 2,319(41.55) \\ \text { 2. (Osteo) artrosis primaria generalizada } & 1,718(30.78) \\ \text { 3. Otras coxartrosis primarias } & 541(9.69) \\ \text { 4. Gonartrosis primaria bilateral } & 137(2.45) \\ \text { 5. Artrosis no especificada } & 128(2.29) \\ \text { 6. Artropatía en la amiloidosis } & 125(2.23) \\ \text { 7. Condromalacia de la rótula } & 119(2.13) \\ \text { 8. Pie plano [pes planus] (adquirido) } & 75(1.34) \\ \text { 9. Hallux rigidus } & 73(1.30) \\ \text { 10. Coxartrosis primaria bilateral } & 34(0.60)\end{array}$

ocuparon los traumatismos en rodillas y piernas desplazando al cuarto lugar los trastornos de los tejidos blandos. Con referencia al segundo grupo el noveno lugar lo ocuparon los trastornos de los nervios, de las raíces y de los plexos nerviosos dejando en décimo lugar a los traumatismos en las caderas y muslos. Al último grupo que tuvo como sexto lugar los traumatismos de los hombros, lo posicionaron en el séptimo lugar los traumatismos de muñecas y manos.

La estación del año que predominó fue otoño con $32.33 \%$ $(3,783.90)$ de las consultas efectuadas, seguida de verano, invierno y primavera (Tabla 5).

Con respecto a la distribución geográfica la mayoría de los pacientes provenían de la Ciudad de México y del Estado de México.

\section{Discusión}

En total se registraron 11,704 consultas externas ortopédicas en el período de Enero a Diciembre de 2017, lo cual es significativo si lo comparamos con un estudio realizado en México en un hospital privado durante cinco años, donde se atendieron 18,780 pacientes. ${ }^{1}$ Esta diferencia entre la cantidad de pacientes atendidos, probablemente se deba a que nuestro estudio se realizó en un hospital de referencia y de acceso general, en comparación con un hospital privado, al cual un muy bajo porcentaje de la población tiene acceso.

Las mujeres tuvieron una prevalencia de $67.17 \%$, siendo mucho mayor que la de los hombres $(32.82 \%)$. Esto coincide con lo reportado en la literatura, donde el sexo femenino tiene dominancia en padecimientos ortopédicos y es incluso un factor de riesgo no modificable de desarrollar patologías como las artrosis. En un metaanálisis se ha demostrado que el riesgo de desarrollar gonartrosis es más elevado en mujeres con un cociente de probabilidades (OR, Odds ratio) combinado de 1.68 (IC95\%): 1.37-2.07). ${ }^{6,7,8}$

El grupo etario al que mayor número de consultas se le otorgaron fue el de la adultez, comprendido por los pacientes en el rango de edades de 40-60 años, lo cual concuerda con lo descrito en la literatura. ${ }^{6,9}$

El motivo principal de consulta externa ortopédica en el hospital de estudio fueron las artropatías, lo cual difiere de lo reportado en otros estudios realizados en nuestro país, ya 
Tabla 4: Principales motivos de consulta externa ortopédica por grupo etario.

Patología
1. Artropatías
2. Dorsopatías
3. Trastornos de los tejidos blandos
4. Traumatismos en rodillas y piernas
5. Traumatismos de codo y antebrazo
6. Traumatismos en muñecas y manos
7. Traumatismos de los hombros
8. Traumatismos en tobillos
9. Traumatismos en las caderas y muslos

Patología

1. Artropatía

4. Traumatismos en rodillas y piernas

Tratismos de los hombros

9. Traumatismos en las caderas y muslos
Adulto joven

1,218
655
$\mathbf{1 4 7}$
225
133
125
97
63
39

Adultez

2,924

1,620

474

290

202

161

152

116

41
Adulto mayor

Total

$\begin{array}{rr}1,439 & 5,581 \\ 723 & 2,998 \\ 208 & 829 \\ 85 & 600 \\ 75 & 410 \\ \mathbf{5 1} & 337 \\ 68 & 317 \\ 40 & 219 \\ 38 & 118\end{array}$

que Echeverría y colaboradores describieron los principales motivos de consulta de primera vez de las cuatro UMAE de Traumatología y Ortopedia, ocupando el primer lugar el síndrome doloroso lumbar o lumbalgia. Un resultado similar se obtuvo en un estudio realizado de 2000 a 2005 en el Hospital Escandón, donde las dorsalgias y lumbociatalgias se posicionaron en el primer lugar., ${ }^{1,10}$

La explicación de lo anterior radica en el incremento de los factores de riesgo modificables de la población de desarrollar artropatías como la obesidad, puesto que México ha experimentado un cambio sustancial en las tasas de este padecimiento, las cuales han ido en aumento en las últimas décadas, posicionando a nuestro país en el cuarto lugar a nivel internacional. En 2006, según la Encuesta Nacional de Salud y Nutrición (ENSANUT), la prevalencia de sobrepeso y obesidad fue de 39.7 y $29.9 \%$ respectivamente. En $2010,32 \%$ de los hombres y $26 \%$ de las mujeres tenían un peso normal. Para 2050 se espera que sólo $12 \%$ de los hombres y $9 \%$ de las mujeres tengan un peso normal, lo que implicaría que el resto de la población padecería sobrepeso u obesidad. ${ }^{11,12,13,14,15}$

La relación de las artropatías como la osteoartrosis y la obesidad se explica porque el aumento del depósito de tejido adiposo provoca una carga mecánica excesiva e incongruente, empeorando la sintomatología de los pacientes. Se sugiere un importante vínculo entre las adiponectinas, la inflamación relacionada con la obesidad y la progresión de la artropatía. ${ }^{6,8,12,13}$

Con referencia a la OA la obesidad y la diabetes mellitus tipo dos (DM2) aumentan significativamente el riesgo de desarrollarla, ya que la OA de rodilla aumenta $15 \%$ por cada aumento de una unidad del IMC. ${ }^{6}$

Es necesario implementar medidas para retrasar la aparición de las artropatías, ya que existen factores de riesgo no modificables como la carga genética, la etnia y el sexo; sin

Tabla 5: Predominio estacional de la consulta externa ortopédica.

$\begin{array}{lcc}\text { Estación } & \mathrm{n}(\%) & \mathrm{p} \\ \text { Primavera } & 1,835.18(15.68) & <0.0001 \\ \text { Verano } & 3,556.84(30.39) & <0.0001 \\ \text { Otoño } & 3,783.90(\mathbf{3 2 . 3 3}) & <0.0001 \\ \text { Invierno } & 2,524.55(21.57) & <0.0001\end{array}$

embargo, al reducir los factores modificables como actividad física, deportes de competición, terapia de reemplazo de estrógenos, alteraciones en la alineación articular (genu varo/valgo), actividades laborales, tabaquismo y la dieta, disminuye la disfuncionalidad, incapacidad y la carga socioeconómica que éstas representan. $8,12,13,16$

La artrosis es la artropatía más común, su carga económica representa de $1-2.5 \%$ del producto interno bruto de los países occidentales y la osteoartrosis es la que representa la mayor parte de esta carga. Esto es preocupante, teniendo en cuenta que en nuestro estudio otras gonartrosis primarias fueron la patología principal del grupo de las artropatías con 2,319 consultas otorgadas, siendo esta patología la principal causa de dolor de rodilla en la población a partir de los 50 años. ${ }^{16,17}$

En general los trastornos musculoesqueléticos son la causa de incapacidad más frecuente en todo el mundo y el grupo poblacional más afectado son los trabajadores. En Europa, 100 millones de ciudadanos padecen dolores musculares y trastornos musculoesqueléticos, incluyendo 40 millones de trabajadores, $25 \%$ de los trabajadores padecen dolor de espalda y $23 \%$ manifiesta padecer dolores musculares. ${ }^{3,18}$

Las lumbalgias son la causa principal de ausentismo laboral en Europa, seguidas de cervicalgia y otros trastornos musculoesqueléticos, posicionándose entre las enfermedades incapacitantes más prevalentes en la población. Además, los trastornos musculoesqueléticos a menudo se acompañan de patologías siquiátricas como depresión y ansiedad, lo que produce mayor retraso a la reincorporación laboral. ${ }^{18}$

Reid y colaboradores reportaron en su estudio que los trastornos musculoesqueléticos son causa importante de incapacidad laboral en Estados Unidos, afectando a 4.1 millones de trabajadores, esto representa 2.1 millones de casos de días de trabajo perdidos. ${ }^{19}$

Es importante tener en cuenta la relación intrínseca entre el tipo de trabajo que se realiza y la aparición de los trastornos musculoesqueléticos, ya que $62 \%$ de los trabajadores de la EU-27 está expuesto durante una cuarta parte del tiempo o más a movimientos repetitivos de manos y brazos, $46 \%$ a posturas dolorosas o extenuantes y $35 \%$ transportan o mueven cargas pesadas.

En Nueva Zelanda el costo económico que tiene el dolor de espalda es de 350 millones, aunado a los 250 millones de 
días de ausencia laboral que tienen los trabajadores a causa de esta patología.

Por lo tanto, conocer los datos estadísticos de los principales motivos de consulta externa ortopédica en México nos permite saber el impacto económico que generan las diversas patologías, la repercusión que tiene en la productividad laboral y es una herramienta para mejorar las condiciones de atención en salud optimizando recursos.

Con respecto al predominio estacional, en nuestro estudio se observó propensión en la estación de otoño, otorgándose $32.3 \%$ de todas las consultas del año. Varios estudios describen la relación de los patrones climáticos con el volumen de la consulta ortopédica por trauma, teniendo una correlación positiva con las temperaturas y negativa con la precipitación; sin embargo, el predominio estacional no está claramente definido por el impacto que tiene el cambio climático en los patrones estacionales de cada región así como las actividades de la población. En Suiza en invierno existe un aumento de la consulta ortopédica por un incremento de los turistas que practican deportes en nieve y sufren algún tipo de traumatismo. ${ }^{20,21,22,23}$

Entonces, se necesitan más estudios que consideren las variaciones climáticas de cada región y las actividades de la población así como la relación del clima con todas las patologías ortopédicas y no únicamente con los casos traumáticos, ya que la información obtenida permitirá una mejor distribución de los recursos para una atención más eficiente de los pacientes. En nuestro país son pocos los datos epidemiológicos que se tienen de las enfermedades musculoesqueléticas, lo que dificulta la optimización de los recursos para la atención adecuada y efectiva de los pacientes en los hospitales.

Entre las limitaciones de nuestro estudio, consideramos que al sólo contar con el registro de atención de un hospital, se puede subestimar el número total de las consultas otorgadas así como las patologías atendidas y la afección que producen en diferentes habitantes del país, además de que existen otros factores intrínsecos de las poblaciones de cada región del país que influyen en la presentación de las enfermedades, los cuales no pudieron ser analizados debido a que nuestra población en su mayoría fueron habitantes de la Ciudad de México y del Estado de México. Otra limitación fue que la base de datos empleada utilizaba diagnósticos que no concordaban con la clasificación estadística internacional de enfermedades y problemas relacionados con la salud CIE-10, por lo que proponemos que todos los hospitales del país empleen una clasificación mexicana o única para catalogar uniformemente los diagnósticos.

Es necesario generar bases de datos de las patologías que padece nuestra población, debido a que nos permitirá analizar las relaciones reales que existen entre las características intrínsecas de nuestra población y la forma de presentación de las enfermedades, lo cual es pilar fundamental para el desarrollo de medidas preventivas que pretendan optimizar la atención del sistema de salud, la disminución de la car- ga económica y la proporción de una mejor calidad de vida para los mexicanos.

En suma, consideramos transcendental que se realicen estudios multicéntricos sobre la epidemiología de las enfermedades musculoesqueléticas en nuestro país, debido a que nos da la pauta para modificar los factores de riesgo y disminuir la presentación de las patologías, además de optimizar los recursos para brindar una atención de calidad a los pacientes. Por lo tanto, sirva este estudio como iniciativa para que más hospitales decidan generar bases de datos y/o hacerlas públicas para su procesamiento y análisis para poder describir la epidemiología de los trastornos musculoesqueléticos en México y establecer medidas de prevención con el fin de disminuir la carga económica y mejorar la calidad de vida de la población.

\section{Conclusión}

Las artropatías, específicamente la gonartrosis, fue el principal motivo de consulta externa, representando casi la mitad de las consultas otorgadas. Hay un predominio de la atención a las mujeres y el rango de edad más comúnmente atendido es de 40-60 años.

\section{Referencias}

1. Rico C. Incidencia de padecimiento ortopédicos en pacientes adultos atendidos en un Hospital de asistencia privada. Acta Ortop Mex. 2007; 21(4): 177-81.

2. Vignolo J, Vacarezza M, Álvarez C, Sosa A. Niveles de atención, de prevención y atención primaria de la salud. Arch Med Int. 2011; 33(1): 11-4.

3. Azcona J, Barrau P, Tapia J, Pardillos J, Ibarz J, Gracia A. Detección precoz de trastornos musculo-esqueléticos: sistema de alertas para la identificación de alta incidencia, correlacionado con poblaciones envejecidas y aplicación de estrategias. Rev Asoc Esp Med Trab. 2016; 25(4): 195-259.

4. Parniapour M, Nordin M, Skovron ML, Frankel VH. Environmentally induced disorders of the musculoskeletal system. Med Clin North Am. 1990; 74(2): 347-59.

5. Harcombe H, McBride D, Derrett S, Gray A. Prevalence and impact of musculoskeletal disorders in New Zealand nurses, postal workes and office workes. Aust N Z J Public Health. 2009; 33(5): 437-41. doi: 10.1111/j.1753-6405.2009.00425.x.

6. Duclos M. Ostearthritis, obesity and type 2 diabetes: the weight of waist circumference. Ann Phys Rehabil Med. 2016; 59(3): 157-60. doi: 10.1016/j.rehab.2016.04.002.

7. Woo J, Lau E, Lee P, Kwok T, Lau WC, Chan C, et al. Impact of osteoarthritis on quality of life in a Hong Kong Chinese population. $J$ Rheumatol. 2004; 31(12): 2433-8.

Wieczorek M, Rat A. Generalidades sobre la artrosis: epidemiología y factores de riesgo. EMC-Aparato Locomotor. 2017; 50(3): 1-12. doi:10.1016/S1286-935X(17)86066-4.

9. Moskowitz R. The burden of osteoarthritis: clinical and quality-of-life issues. Am J Manage Care. 2009; 15(8): S223-9.

10. Bárcena L, De la Fuente J, Tamez J, Salvatori J. Análisis de la demanda de atención y epidemiología. En: Paiz J, Cruz F, Fajardo G, Navarro F, Carrillo R. Traumatología y ortopedia. México, D.F.: Editorial Alfil; 2013. 7-24.

11. Davis K, Kotowski S. Prevalence of musculoskeletal disorders for nurses in hospitals, long-term care facilities, and home health care: a comprehensive review. Hum Factors. 2015; 57(5): 754-92. doi: $10.1177 / 0018720815581933$. 
12. Palazzo C, Nguyen C, Lefevre-Colau MM, Rannou F, Poiraudeau S. Risk factors and burden of osteoarthritis. Ann Phys Rehabil Med. 2016; 59(3): 134-8. doi: 10.1016/j.rehab.2016.01.006.

13. Frye C, Shmalberg J, Wakshlag JJ. Obesity, exercise and orthopedic disease. Vet Clin North Am Small Anim Pract. 2016; 46(5): 831-41. doi: 10.1016/j.cvsm.2016.04.006.

14. Rtveladze K, Marsh T, Sanchez L, Levy D, Melendez G, Webber L, et al. Obesity prevalence in Mexico: impact on health and economic burden. Public Health Nutr. 2014; 17(1): 233-9. doi: 10.1017/ S1368980013000086.

15. Baquera S, Campos I, Rojas R, Rivera J. Obesidad en México: epidemiología y políticas de salud para su control y prevención. Gac Med Mex. 2010; 146: 397-407.

16. Bierman S, Koes B. Risk factors and prognostic factors of hip and knee osteoarthritits. Nat Clin Pract Rheumatol. 2006; 3(2): 78-85. doi: 10.1038/ncprheum0423.

17. Nguyen U, Zhang Y, Niu J, Zhang B, Felson D. Increasing prevalence of knee pain and symptomatic knee osteoarthritis: survey and cohort data. Ann Intern Med. 2011; 155(11): 725-32. doi: 10.7326/00034819-155-11-201112060-00004.
18. Bevan S. Economic impact of musculoskeletal disorders (MSDs) on work in Europe. Best Pract Res Clin Rheumatol. 2015; 29(3): 356-7. doi: 10.1016/j.berh.2015.08.002.

19. Reid C, Bush P, Cummings N, McMullin D, Durrani S. A review of occupation knee disorders. J Occup Rehabil. 2010; 20(4): 489-501. doi: 10.1007/s10926-010-9242-8.

20. Livingston K, Miller P, Lierhaus A, Matheney T, Mahan S. Does weather matter? The effect of weather patterns and temporal factors on pediatric orthopedic trauma volume. Open Orthop J. 2016; 10: $550-8$.

21. Rising W, O'Daniel J, Roberts C. Correlating weather and trauma admissions at a level I trauma center. J Trauma. 2006; 60(5): 10961100. doi: 10.1097/01.ta.0000197435.82141.27.

22. Ali A, Willett $\mathrm{K}$. What is the effect of the weather on trauma workload? A systematic review of the literature. Injury. 2015; 46(6): 945-53. doi: 10.1016/j.injury.2015.03.016.

23. Matter K, Widmer M, Busato A. Seasonal variation in orthopedic health services utilization in Switzerland: the impact of wintersport tourism. BMC Health Serv Res. 2006; 6: 25. doi: 10.1186/1472-69636-25. 